\title{
"Osteomyelitis Of Mandible"-A Rare Presentation Of Osteopetrosis
}

\author{
I AHMAD, SZ ABBAS, F HAQUE, M RASHID, SA AHMAD
}

\begin{abstract}
Osteopetrosis is a rare metabolic disease. Dental abnormalities may be attributed to the pathological changes in osteopetrosis. Patients with disease seem to be especially susceptible to osteomyelitis of mandible. A 9 yrs old girl presented with complaints of jaw swelling on left side with multiple discharging sinuses since last seven months. The radiograph of left mandible (oblique lateral view) showed periodontal involvement of associated molars with loss of lamina dura with small sequestrum with irregularity and erosions of the mandibular cortical margins. The patient was diagnosed as a case of osteopetrosis with superadded mandibular osteomyelitis.
\end{abstract}

Ind J Radiol Imag 2006 16:2:253-256

\section{Key words : - Osteopetrosis, Osteomyelitis}

\section{Introduction}

Osteopetrosis (marble bone disease or Albers Schonberg disease) is a rare metabolic disease. It may be seen with numerable disorders referable to head and neck. It occurs both in benign or malignant forms. It results in abnormal bone development associated with complications like blindness, stunted growth, abnormal tooth development and fragile bones. Dental abnormalities may be attributed to the pathological changes in osteopetrosis. Patients with disease seem to be especially susceptible to osteomyelitis of mandible. We present a case of osteopetrosis with mandibular osteomyelitis with the purpose to review the entity and to reemphasize an important, less obvious, clinical presentation.

\section{Case Report}

A 9 yrs old girl presented with complaints of jaw swelling on left side with multiple discharging sinuses since last seven months. There was no history of any blood disorder in family, hematemesis, jaundice, chronic fever, loss of weight, any visual or hearing problem. There was history of fracture of left thigh bone after trivial trauma two years back.

On general examination there was moderate pallor,few significant enlarged submandibular and lateral cervical lymph nodes were found. There was mild hepatosplenomegaly (liver span 14cms,spleen 12cms) . Vision and fundi were normal. There was no hearing defect. On local examination multiple discharging sinuses were seen on left side of the mandible with yellowish puspoints and tender jaw . Intraoral examination showed pale oral mucosa and generalized periodontitis; there was malocclusion of teeth. A hard exuburent, non tender swelling was noticed on lateral aspect of left thigh, however the overlying skin was normal.

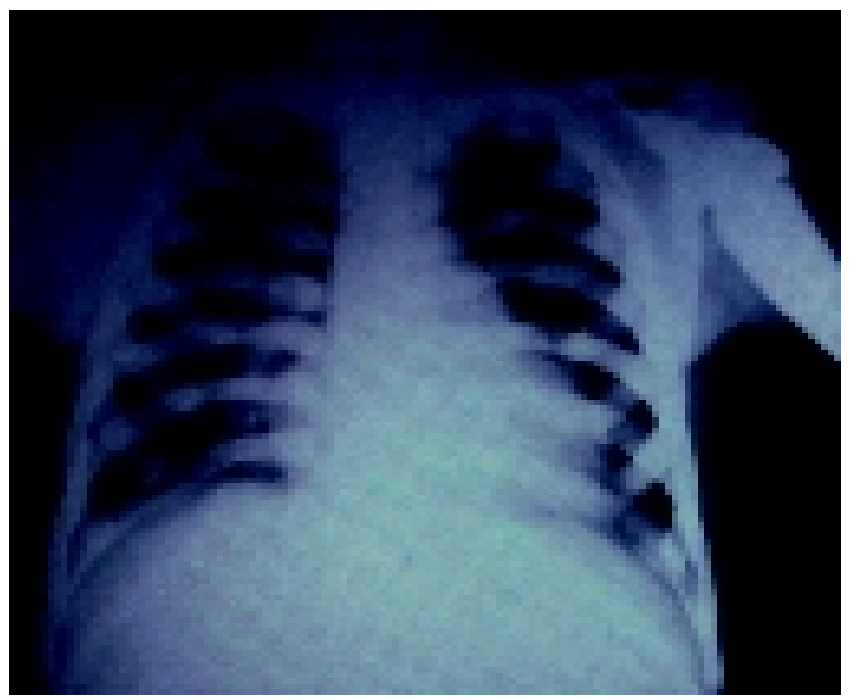

Fig.1 : The chest radiograph showed increased bone density with bilateral clear lung fields

From the Dept. of Radiodiagnosis, JN Medical College , AMU, Aligarh,India.

Request for Reprints: Dr. S.Zafar Abbas, Department of Radiodiagnosis, J.N.M.C., A.M.U, Aligarh - 202002

Received 20 November 2005; Accepted 10 April 2006 
254 I Ahmad et al

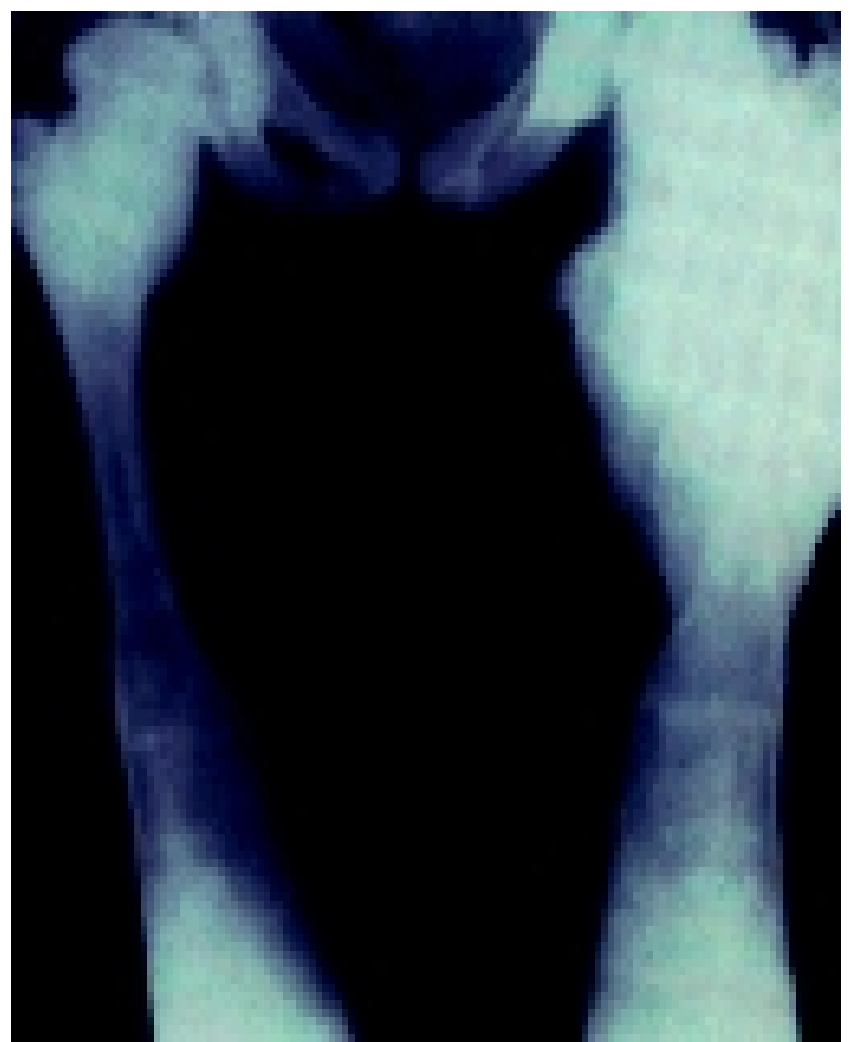

Fig.2 : The radiograph of pelvis with both thigh revealed revealed old healed fracture of left femur with excessive callus formation with Erlenmeyer flask deformity with generalized increased bone density

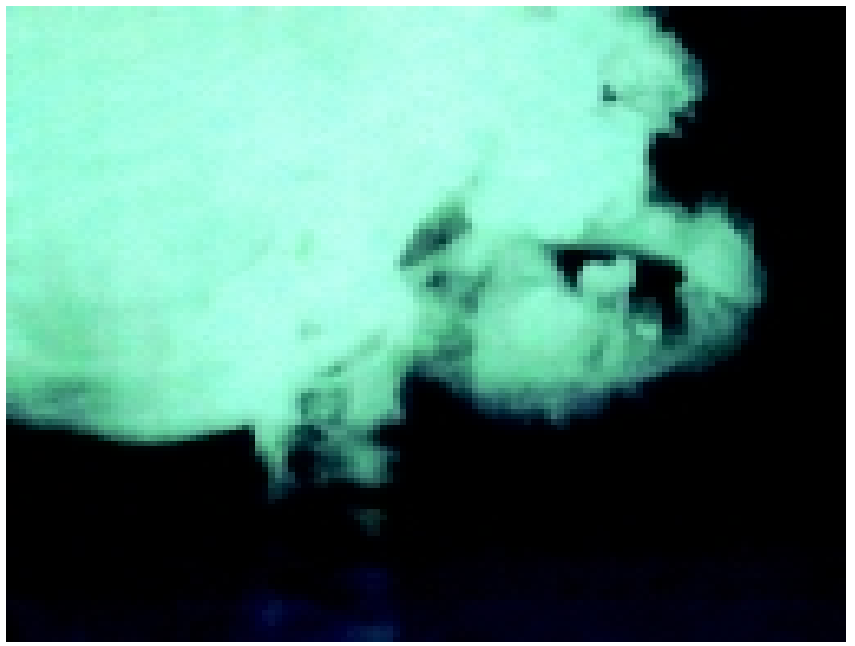

Fig 3 : The radiograph of left mandible(oblique lateral view) showed periodontal involvement of associated molars with loss of lamina dura with small sequestrum with irregularity and erosions of the mandibular cortical margins.

Laboratory test revealed $\mathrm{Hb} 9 \mathrm{mg} \%$, WBC 2.3X109/L, DLC showed neutrophils $70 \%$ and lymphocytes $30 \%$. Serum chemistry revealed Cl 106 mg/l, HCo3 $21 \mathrm{mg} / \mathrm{l}$, Ca $9.3 \mathrm{mg} / \mathrm{dl}$ and alkaline phosphatase $8.0 \mathrm{KA}$ unit/dl.

The chest radiograph showed increased bone density with bilateral clear lung fields. The radiograph of pelvis with both thighs revealed old healed fracture of left femur with excessive callus formation with Erlenmeyer flask deformity with generalized increased bone density. The radiograph of left mandible (oblique lateral view) showed periodontal involvement of associated molars with loss of lamina dura with small sequestrum with irregularity and erosions of the mandibular cortical margins. The patient was diagnosed as a case of osteopetrosis with superadded mandibular osteomyelitis.

As the facility of hyperbaric oxygen therapy was not available in our setup, patient was advised hemimandibulectomy with reconstruction surgery, which her parents refused. So the patient was put on systemic antibiotic therapy and local debridement of affected jaw was done.

\section{Discussion}

Osteopetrosis is a rare metabolic bone disease characterized by a generalized increase in skeletal mass. This inherited disorder results from a congenital defects in the development or function of the osteoclast(1).

The consequent impairment of bone resorption prevents formation of bone marrow cavities. The pathogenetic defect may be intrinsic either to the osteoclast lineage or to the mesenchymal cells that constitute the microenvironment supporting the development and activation of osteoclasts. Three clinically distinct forms of osteopetrosis are recognized-infantile malignant autosomal recessive form, fatal within first few years of life (in the absence of effective therapy); intermediate autosomal recessive form, appears during the first decade of life but does not follow the malignant course and the adult benign dominant form with full life expectancy but many orthopedic problems. The disease represents a spectrum of clinical variants because of the heterogenecity of genetic defects resulting in osteoclast dysfunction(2).Osteopetrosis complicated by the development of osteosarcoma has been reported(3). Survival after $20 \mathrm{yrs}$ of age is rare.

Orthopedic problems in the intermediate and autosomal dominant forms include bone pain ( $26 \%$ of patients), increased fractures (40\%) coxa vara, long bone bowing, hip and knee degenerative arthritis and osteomyletis. Dental abnormalities may be attributed to the pathological changes in osteopetrosis. Patients with the disease seem to be specially susceptible to caries. Constriction of 
canals housing neurovascular bundles that supply teeth and jaws, along with obliteration of the marrow cavities and the dental pulp chambers, is the most likely contributing factor to bone necrosis and dental caries(4).Other dental changes may include delayed eruption and early loss of teeth, enamel hypoplasia,malformed roots and crowns and thickening of the lamina dura.(5) Osteomyelitis, due to dental caries $(10 \%)$, is well recognized hazard in osteopetrosis due to reduced blood circulation to bone as a result of obliteration and fibrosis of the marrow. It is a potentially severe infection that runs a protracted course, due to the accompanying severe anemia and neutropenia. (6)

Radiography show uniform increase bone density without corticomedullary demarcation. The long bones have a dense chalk like appearance. They may have an 'Erlenmeyer flask' deformity at their ends due to failure of metaphyseal remodelling, giving gross distal under tubulation, and the presence of dense bone, vertical fine lucencies extending to the metaphysis are present probably due to vascular channels being better seen against dense bone. Fractures are usually transverse and heal with normal callus. Bowing of the long bones as well as coxa vara may be present due to multiple fractures.

There is also an increase in density of bone at the base of skull especially prominence in the floor of anterior cranial fossa. The sphenoid and frontal sinuses and mastoids may be underpneumatised. Neural foramina may be encroached upon.

The vertebral column has a "sandwich" or "ruggerjersey" appearance with dense sclerotic bone at each end plate of the vertebral body. Spondylosis of the lumbar spine has been reported(7).

A 'bone within a bone' or endobone phenomenon may be seen in small bones of the hands but, with increased density around the periphery.

The most common complication of the osteopetrosis is pathologic fractures, those with congenital presentation are likely to have the most fractures. Femoral shaft fractures either with transverse or short oblique pattern are most common. Other common locations are inferior neck of femur and posterior tibia. Upper extremity fractures are also reported. fracture healing seems to occur at a normal rate but the onset of callus formation after injury is variable(8). Nonunion \& delayed union of fraction may occur.

Management of the patients with osteopetrosis requires a comprehensive approach to characteristic clinical problems including hematological and metabolic abnormalities, fractures, deformity back pain, bone pain, osteomyelitis and neurological sequalae. Medical management of osteopetrosis is based on efforts to stimulate host osteoclasts on provide in alternate source of osteoclasts. Stimulation of host osteoclast has been attempted with calcium restriction, calcitriol, steroids, parathyroid hormone and interferon. Hyperbaric oxygen has been shown to be beneficial in the treatment of mandibular osteomyelitis(9) .It has a bactericidal and bacteriostatic effect in vitro and in vivo but the bone infection is not the only factor but reduced tissue resistance due to avascularity of the marrow spaces and the partial obliteration of the mandibular canal appeared to be the basic problem. The beneficial effect is probably resulted from the improved vascular supply and increased oxygen perfusion to the ischaemic areas of infection(10). Bone marrow transplant has been used with cure for infantile malignant osteopetrosis. As osteopetrosis likely represents a spectrum of underlying etiologies resulting in osteoclast dysfunction, effective therapies need to be individualized.

\section{References}

1. Felix R, Hofstetter W, Cecchini MG.Recent developments in the pathophysiology of osteopetrosis.Indian Pediatrics 2003 jun;40(6):561-5.

2. Shapiro F.Osteopetrosis.current clinical considerations. Clin Orthop 1993 sep;(294):87-97.

3. Fasth A,Porras O.Human malignant osteopetrosis: pathophysiology,mangement and the role of bone marrow transplantation.Pediatr transplant.1993;3 suppl 1:102-7.

4. Hinds EC.Non inflammatory bone disease.J. Oral Surg 1970 jan;28:27.

5. DickHM,Simpson J.Dental changes in osteopetrosis. Oral Surg 1972 sept;34:408.

6. Lawoyin DO,Daramola JO,Ajabge HA,Nyako EA, Lawoyin JO.Osyeomyelitis of the mandible associated with osteopetrosis:Report of a case. $\mathrm{Br} \mathrm{J}$ of oral and max surg.1988;26:330-5.

7. Benichow OD,Laredo JD, Deverenejoul MC. Type II 
autosomal osteopetrosis.Bone.2000jan;26(1):87-93.

8. Stoker DJ.Osteopetrosis.Semin musculoskel radio.2002dec;6(4):299-305.

9. Mainous EG,Boyne PJ,Hart GB.Hyperbaric oxygen treatment of mandibular osteomyelitis:Report of three cases.J am dent assoc1973;87:1426.

10. Mainous EJ,Hart GB,Soffa DJ,Graham GA,. Hyperbaric oxygen treatment of mandibular osteomyelitis in osteopetrosis.J oral surg 1975 april;33:288-291. 